\title{
A Novel ECM1 Splice Site Mutation in Lipoid Proteinosis: Case Report plus Review of the Literature
}

\author{
Linda K. Rey ${ }^{a, c}$ Jürgen Kohlhase ${ }^{d}$ Katrin Möllenhoff ${ }^{b, c}$ Gabriele Dekomien ${ }^{a, c}$ \\ Jörg T. Epplen ${ }^{a, c, e}$ Sabine Hoffjan ${ }^{a, c}$ \\ Departments of a Human Genetics and ${ }^{b}$ Dermatology, Venereology and Allergology, Ruhr-University Bochum, and \\ ${ }^{c}$ Center for Rare Diseases Ruhr (CeSER), Bochum, dCenter for Human Genetics, Freiburg, and eFaculty of Health, \\ University Witten/Herdecke, Witten, Germany
}

\section{Key Words}

ECM1 - Genodermatosis · Lipoid proteinosis · Splice site mutation · Urbach-Wiethe syndrome

\begin{abstract}
Lipoid proteinosis (LP) is an autosomal recessive genodermatosis known to be caused by mutations in ECM1. Nonsense and missense mutations are the most common variations in LP. Up to date, only 6 splice site mutations have been observed. We report on a 26 -year-old female LP patient from a Turkish consanguineous family carrying a novel homozygous splice site mutation in intron 8 of the ECM1 gene and summarize the current knowledge on ECM1 mutations and possible genotype-phenotype correlations.
\end{abstract}

(C) 2016 S. Karger AG, Basel

Lipoid proteinosis (LP) is a rare autosomal recessive disease (OMIM 247100) caused by mutations in the extracellular matrix protein 1 (ECM1) gene in chromosome 1q21 [Hamada et al., 2002]. It is characterized by hoarseness of voice, a scar-like skin involvement and yellowish infiltrations of mucous membrane. Histological findings show abnormal lipid and protein (hyaline) accumulation in various tissues. Accordingly, it is often referred to as hyalinosis cutis et mucosae. Urbach-Wiethe syndrome is another synonym used, as Camillo Wiethe and Erich Urbach were the first authors to describe the symptoms in 1929 [Urbach and Wiethe, 1929]. Symptoms usually develop within the first year of life, typically in infants with a hoarse cry as vocal cords are infiltrated first. Skin lacerations develop later in childhood [Hamada, 2002], but severity of skin involvement and other symptoms may be variable [Hamada et al., 2002]. Neuropsychiatric abnormalities and seizures are occasionally seen in patients with LP as well as calcifications of the amygdala and the temporal lobe in MR imaging. Altogether, the disease course is slowly progressive with normal life expectancy [Hamada et al., 2002]. Up to date, there are more than 50 reported mutations in ECM1 associated with LP which mainly include nonsense and missense mutations [Chan et al., 2007]. Only 6 splice mutations are so far listed in the database (HGMD Professional 2015.3, http://www. hgmd.cf.ac.uk/ac/index.php). Here, we report on a female patient from a consanguineous Turkish family who

\section{KARGER}

E-Mail karger@karger.com www.karger.com/msy
(C) 2016 S. Karger AG, Basel

$1661-8769 / 16 / 0071-0026 \$ 39.50 / 0$
Dr. Linda K. Rey

Department of Human Genetics, Ruhr-University Bochum

Universitätsstrasse 150

DE-44801 Bochum (Germany)

E-Mail linda.rey@ rub.de 


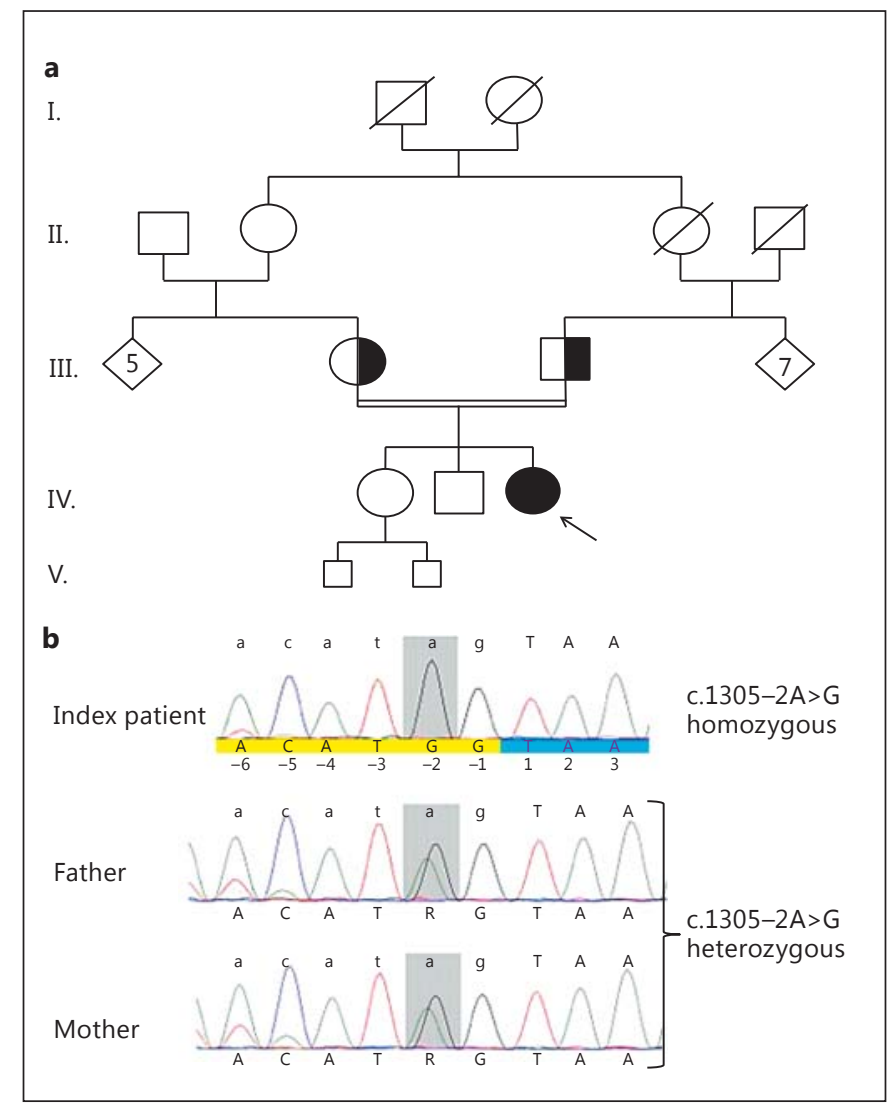

Fig. 1. a Pedigree of the family. Black symbol: affected member; semi-black symbol: unaffected carrier; white symbol: unaffected individual. b Sequence analyses in the index patient and her parents.

carries a novel homozygous splice site mutation in the ECM1 gene and summarize the current knowledge on ECM1 mutations and possible genotype-phenotype correlations.

\section{Patient and Methods}

\section{Case Report}

Our patient is a 26-year-old female of Turkish decent, born to consanguineous parents (first-degree cousins; see fig. 1a). She has 2 unaffected siblings, 32 and 30 years of age. First symptoms occurred in infancy beginning with an almost inaudible and hoarse cry. Recurrent itchy and hyperkeratotic papules appeared on the upper back during the first few months, spreading to the face and the extremities in the following period of time. Speech was difficult to understand due to the hoarse and low voice. A slight delay of speech development was noted at age 5.5. At the age of 6 years, the patient was diagnosed with lipoid proteinosis, and the case was presented at a German dermatology convention in 1994 [U. Theis-
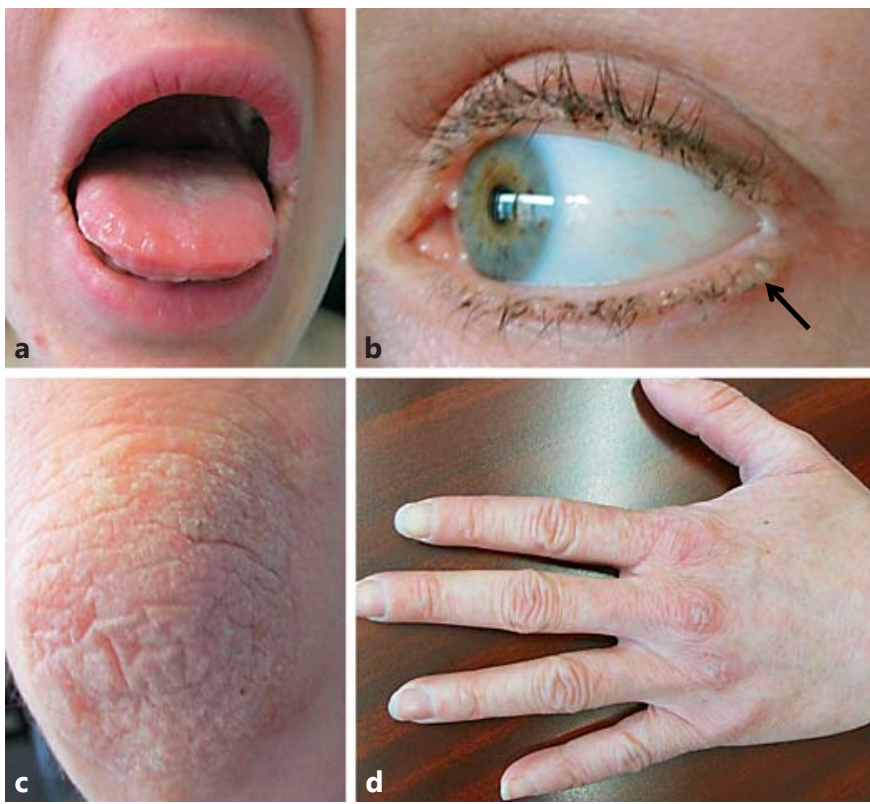

Fig. 2. Clinical findings in the index patient at age 26. a Thickened mucosa of the tongue. $\mathbf{b}$ Moniliform blepharosis, yellow nodules on upper and lower eyelid margin (arrow). c Hyperkeratosis of the elbow. d Hyperkeratosis and xerosis of the hand.

sen, pers. commun.]. A first microlaryngoscopy was performed at the age of 7, and a biopsy of the epiglottis was taken which showed chronic inflammation of the squamous tissue. No information about hyaline material and PAS staining was provided. In yearly performed laryngoscopies, thickening of the vocal cords was observed and treated by laser extirpation. Neurological abnormalities were not reported in our patient, especially not any kind of seizures. However, neuropsychological evaluation at 17 years of age revealed slight deficits in affect interpretation and visual memory construction. An MRI scan at the age of 21 showed a normal result without calcifications of the temporal lobes. Cardiac examination was unremarkable. Recently, a tonsillectomy was performed due to chronic infection and dysphagia.

Current clinical investigation (fig. 2) revealed hypertrophy and induration of the tongue with yellow plaques and papules. Movement of the tongue was limited due to thickening of the frenulum. Skin-toned papules were seen on the margin of the lower eye lid (moniliform blepharosis). Knees, elbows, axillar and genital region presented with white to yellow papules. A generalized xerosis cutis was apparent. Overall, skin manifestations in our patient were rather subtle apart from tongue and eyelid involvement. The voice of the patient is still remarkably hoarse.

\section{Molecular Genetic Analysis}

Sanger sequencing of all exons and adjacent intronic sections of the ECM1 gene in our patient identified the homozygous base exchange c.1305-2A>G in intron 8, which causes an alteration of the splice acceptor site (fig. 1b). Both unaffected parents were confirmed as heterozygous carriers. 


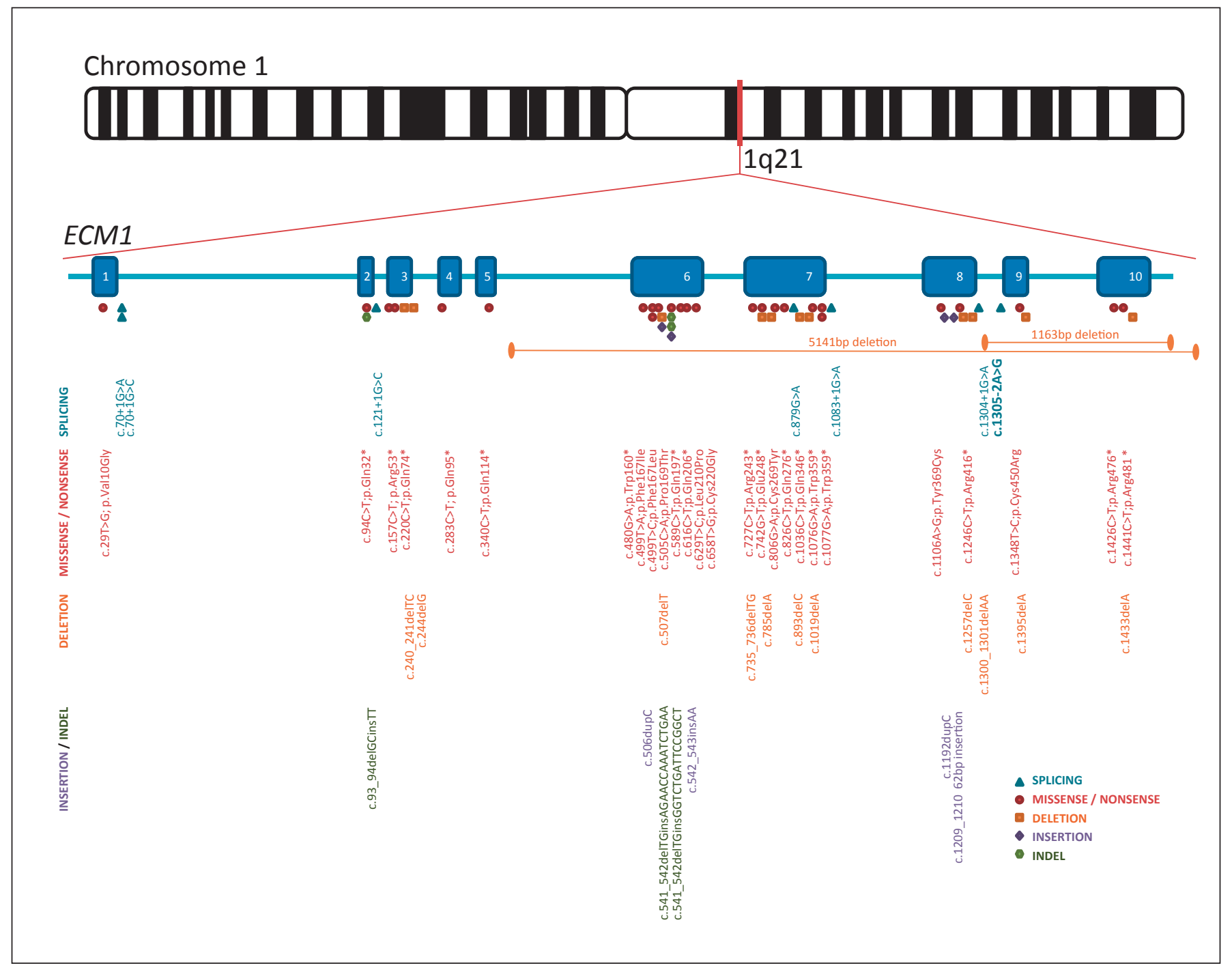

Fig. 3. Mutations in ECM1 as published in HGMD and the literature.

\section{ECM1 Mutations in LP: Review of the Literature and Discussion of Possible Genotype-Phenotype Correlations}

\section{Mutational Spectrum}

The ECM1 gene is located in chromosome 1q21 and contains 10 exons [Smits et al., 1997]. Figure 3 gives an overview of the ECM1 mutations known to date. The majority of patients were found to carry nonsense and missense mutations in exons 6 and 7 [Nasir et al., 2011], but mutations in all 10 exons have been described. LP, as an autosomal recessive disease, is more common in consanguineous families, and most families carry unique muta- tions except for South-African patients, where a founder effect with one common ancestral allele has been suggested. South Africa has the largest group of LP patients worldwide, and most of them are homozygous for a nonsense mutation (p.Q276X) in exon 7 [Van HougenhouckTulleken et al., 2004]. Recently, a common ancestral allele has been suggested in Chinese LP patients as well; 7 of 8 reported patients in China were homozygous or at least compound heterozygous for the missense mutation p.C220G in exon 6 [Wang CY et al., 2006; Wang XP et al., 2009; Zhang et al., 2014]. Splice site mutations have rarely been observed so far. 
Table 1. Clinical information for the published patients carrying splice site mutations

\begin{tabular}{|c|c|c|c|c|c|c|c|}
\hline & $\begin{array}{l}\text { Horev et al., } \\
{[2005]}\end{array}$ & $\begin{array}{l}\text { Chan et al. } \\
\text { [2007] }\end{array}$ & $\begin{array}{l}\text { Chan et al. } \\
\text { [2007] }\end{array}$ & $\begin{array}{l}\text { Chan et al. } \\
\text { [2007] }\end{array}$ & $\begin{array}{l}\text { Abbas et al. } \\
{[2013]}\end{array}$ & $\begin{array}{l}\text { Gao et al. } \\
{[2013]}\end{array}$ & Our patient \\
\hline \multicolumn{8}{|l|}{ Genetics } \\
\hline Mutation & c. $70+1 \mathrm{G}>\mathrm{C}$ & c. $70+1 \mathrm{G}>\mathrm{A}$ & c. $1083+1 \mathrm{G}>\mathrm{A}$ & c. $1304+1 \mathrm{G}>\mathrm{A}$ & c. $121+1 \mathrm{G}>\mathrm{C}$ & c. $879 \mathrm{G}>\mathrm{A}$ & c. $1305-2 \mathrm{~A}>\mathrm{G}$ \\
\hline \multirow[t]{2}{*}{ Location } & intron 1 & intron 1 & intron 7 & intron 8 & intron 2 & exon 7 & intron 8 \\
\hline & homozygous & $\begin{array}{l}\text { compound- } \\
\text { heterozygous }\end{array}$ & homozygous & homozygous & homozygous & $\begin{array}{l}\text { compound- } \\
\text { heterozygous }\end{array}$ & homozygous \\
\hline Consanguinity & + & n.a. & n.a. & n.a. & + & - & + \\
\hline 2nd mutation & - & p.V10G & - & - & - & p.P169T & - \\
\hline \multicolumn{8}{|l|}{ Patients } \\
\hline Gender & $\mathrm{f} / \mathrm{m} / \mathrm{f}$ & n.a. & n.a. & n.a. & $\mathrm{f}$ & f & $\mathrm{f}$ \\
\hline Age & $18 / 10 / 1.5$ ys & n.a. & n.a. & n.a & 8 ys & 20 ys & 26 ys \\
\hline Origin & Israel (Arab) & Poland & Pakistan & Pakistan & Lebanon & China & Turkey \\
\hline \multicolumn{8}{|l|}{ Clinical features } \\
\hline Moniliform blepharosis & $+1+1-$ & n.a. & n.a. & n.a. & + & + & + \\
\hline Thickened oral mucosa & $+1+1-$ & n.a. & n.a. & n.a. & + & + & + \\
\hline cMRI/cCT abnormalities & n.a. & n.a. & n.a. & n.a. & - & n.a. & - \\
\hline Hoarseness & $+/+1+$ & n.a. & n.a. & n.a. & + & n.a. & + \\
\hline Hyperkeratotic plaques & $+1+1-$ & n.a. & n.a. & n.a. & + & n.a. & + \\
\hline Scarring & $+/+/-$ & n.a. & n.a. & n.a & + & n.a. & - \\
\hline
\end{tabular}

$\mathrm{f}=$ Female; $\mathrm{m}=$ male; n.a. = not available; $\mathrm{ys}=$ years; $+=$ yes/present $;-=$ no/not present.

\section{Splice Variations}

There are 3 known ECM1 splice variants (ECM1a, ECM1b, ECM1c) and an additional fourth splice variant coding for proteins which contain $540,415,559$, and 57 amino acids, respectively [Horev et al., 2005]. ECM1a consists of all 10 exons and is mainly found in basal keratinocytes, dermal blood vessels, placenta and heart tissue but also in various other tissues as it is the most commonly expressed splice variant, whereas ECM1b lacks exon 7 and is restricted to expression in tonsils and keratinocytes [Chan et al., 2007]. ECM1c contains an alternative exon $5 \mathrm{a}$. Its expression pattern might be restricted to the basal layer of the epidermis but is still mainly unknown as well as the expression of the fourth splice variant [Sercu et al., 2008]. Our reported novel splice site mutation probably influences all 3 known splice variants ECM1a, b and c, as it is located in intron 8 and presumably leads to alteration of the splice acceptor site, which may result in complete or incomplete exon skipping.

Of the 6 splicing mutations in ECM1 reported so far, 2 were located in intron 1 [Chan et al., 2007; Horev et al., 2009] and one each in intron 2 [Abbas et al. 2013], intron 7, intron 8 [Chan et al., 2007], and in exon 7 [Gao et al., 2013]. All of them potentially lead to an alteration in splice donor sites and exon skipping and as a result of a shortened, nonfunctional protein. However, only a few were functionally tested [Horev et al., 2005; Gao et al., 2013]. The novel splice site mutation in intron 8 in our patient is the first described mutation at the $5^{\prime}$ end of an intron, presumably resulting in a cryptic splice acceptor site. Unfortunately, we were unable to obtain tissue samples for functional analyses.

Clinical information (table 1) was provided for 3 out of the 6 published splice site mutations [Horev et al., 2005; Abbas et al., 2013; Gao et al., 2013]. All patients showed beaded papules on the lower and upper eyelid margin (moniliform blepharosis), hyperkeratotic plaques and thickening of the oral mucosa except for the youngest patient reported [Horev et al., 2005]. As this patient was just 1.5 years old at the time of publication, it is likely that symptoms will develop later on in life. Hoarseness of voice was present in all patients. Unfortunately, information on cranial imaging was not provided except for our patient and one other case [Abbas et al., 2013]. Both were unremarkable and showed no calcifications. At the moment, the number of published cases with splice site mutations is insufficient for determining whether there is a genotype-phenotype correlation for these mutations. A previous study by Hamada et al. [2003] suggested a genotype-phenotype correlation based on the location of the mutation, with a slightly milder phenotype for patients with mutations in exon 7 compared to patients with mutations outside exon 7 . The splice variant ECM1b lacks exon 7 and is therefore not affected by mutations located in this exon. However, a recent study by Youssefian et al. [2015] noted phenotypic heterogeneity in 3 families with $12 \mathrm{LP}$ patients, although all patients were homozygous for the mutation c.507delT in exon 6 . While 
existing features of LP were similar in most patients, severity and expressivity differed substantially between patients, even between patients from the same family.

\section{Functional Role of ECM1}

The exact underlying pathomechanism of LP and ECM1's role in it is still unknown. ECM1 encodes an 85$\mathrm{kDa}$ protein which plays an important role in skin physiology [Chan, 2004]. For example, it is considered to act as 'biological glue' in various tissues [Chan, 2004]. Interestingly, LP is the only monogenetic disease associated with ECM1 mutations so far, and no genetic heterogeneity has been observed in LP [Chan et al., 2007].

Apart from its functional role in skin physiology, ECM1 has been implicated in tumorigenesis and tumor metastasis [Sercu et al., 2008; Lee et al., 2015], mainly due to its promotion of blood vessel proliferation and its role in angiogenesis [Chan et al., 2007]. Studies have shown that expression of ECM1 is increased in most epithelial tumors such as breast cancer, lung cancer, gastric cancer, and colon adenocarcinomas [Sercu et al., 2008]. ECM1 expression was also shown to be a prognostic marker for poor long-term survival in breast cancer patients [Lal et al., 2009]. ECM1 expression further correlates with metastasis of the tumor, as metastasized breast cancer was more likely to be ECM1 positive than those without metastases [Wang et al., 2003]. In addition to its role in tumorigenesis, ECM1 was implicated in the regulation of intestinal permeability and suggested as a potential risk factor for ulcerative colitis and other chronic inflammatory bowel diseases, though its exact functional role remains unknown [McCole, 2014].

\section{Conclusion}

In this study, we present a novel splice site mutation, bringing the count to 7 splice site mutations and a total of 54 mutations in the ECM1 gene. Recent reports - in contrast to former observations - argue against an obvious genotype-phenotype correlation and suggest that intraand interfamilial variability in severity and expression of LP seems more likely. Analysis of additional patients, also with the use of modern sequencing technologies (NGS), will certainly further increase our knowledge on genotype-phenotype correlations and reveal whether mutations in additional genes may also be involved in the pathogenesis of LP.

\section{Statement of Ethics}

Clinical and genetic evaluation of the presented patient was part of the routine diagnostic procedure. The patient and all family members gave written informed consent to the analyses as well as the publication of the results.

\section{Disclosure Statement}

The authors declare that there is no conflict of interests.

\section{References}

Abbas O, Farooq M, El Khoury J, Kibbi AG, Fujikawa $\mathrm{H}$, et al: A novel splice-site ECM1 gene mutation in a Lebanese girl with lipoid proteinosis. Int J Dermatol 52:824-826 (2013).

Chan I: The role of extracellular matrix protein 1 in human skin. Clin Exp Dermatol 29:52-56 (2004).

-Chan I, Liu L, Hamada T, Sethuraman G, McGrath JA: The molecular basis of lipoid proteinosis: mutations in extracellular matrix protein 1. Exp Dermatol 16:881-890 (2007).

Gao D, Lian P, Wang R, Zhang L, Wang X, Chen $\mathrm{J}$ : Identification of a novel splicing mutation of ECM1 in a rare lipoid proteinosis family. J Dermatol 40:675-677 (2013).

Hamada T: Lipoid proteinosis. Clin Exp Dermatol 27:624-629 (2002).
Hamada T, McLean WH, Ramsay M, Ashton GH, Nanda A, et al: Lipoid proteinosis maps to $1 \mathrm{q} 21$ and is caused by mutations in the extracellular matrix protein 1 gene (ECM1). Hum Mol Genet 11:833-840 (2002).

Hamada T, Wessagowit V, South AP, Ashton GH, Chan I, et al: Extracellular matrix protein 1 gene (ECM1) mutations in lipoid proteinosis and genotype-phenotype correlation. J Invest Dermatol 120:345-350 (2003).

Horev L, Potikha T, Ayalon S, Molho-Pessach V, Ingber A, et al: A novel splice-site mutation in $E C M-1$ gene in a consanguineous family with lipoid proteinosis. Exp Dermatol 14:891-897 (2005).
Horev L, Wollina DU, Potikha T, Hafner A, Ingber A, et al: Lipoid proteinosis: identification of two novel mutations in the human ECM-1 gene and lack of genotype-phenotype correlation. Acta Derm Venereol 89:528-529 (2009).

Lal G, Hashimi S, Smith BJ, Lynch CF, Zhang L, et al: Extracellular matrix 1 (ECM1) expression is a novel prognostic marker for poor long-term survival in breast cancer: a Hospital-based Cohort Study in Iowa. Ann Surg Oncol 16:2280-2287 (2009).

Lee KM, Nam K, Oh S, Lim J, Kim RK, et al: ECM1 regulates tumor metastasis and CSC-like property through stabilization of betacatenin. Oncogene 34:6055-6065 (2015).

McCole DF: IBD candidate genes and intestinal barrier regulation. Inflamm Bowel Dis 20: 1829-1849 (2014).
30
Rey/Kohlhase/Möllenhoff/Dekomien/ Epplen/Hoffjan 
Nasir M, Latif A, Ajmal M, Qamar R, Naeem M, Hameed A: Molecular analysis of lipoid proteinosis: identification of a novel nonsense mutation in the ECM1 gene in a Pakistani family. Diagn Pathol 6:69 (2011).

-Sercu S, Zhang L, Merregaert J: The extracellular matrix protein 1: its molecular interaction and implication in tumor progression. Cancer Invest 26:375-384 (2008).

Smits P, Ni J, Feng P, Wauters J, Van Hul W, et al: The human extracellular matrix gene 1 (ECM1): genomic structure, cDNA cloning, expression pattern, and chromosomal localization. Genomics 45:487-495 (1997).
Urbach E, Wiethe C: Lipoidosis cutis et mucosae. Virchows Arch Path Anat 273:285-319 (1929).

Van Hougenhouck-Tulleken W, Chan I, Hamada $\mathrm{T}$, Thornton H, Jenkins T, et al: Clinical and molecular characterization of lipoid proteinosis in Namaqualand, South Africa. Br J Dermatol 151:413-423 (2004).

-Wang CY, Zhang PZ, Zhang FR, Liu J, Tian HQ, Yu L: New compound heterozygous mutations in a Chinese family with lipoid proteinosis. Br J Dermatol 155:470-472 (2006).

Wang L, Yu J, Ni J, Xu XM, Wang J, et al: Extracellular matrix protein 1 (ECM1) is over-expressed in malignant epithelial tumors. Cancer Lett 200:57-67 (2003).
-Wang XP, Huo J, Liu Y, Wang WJ, Xu QQ, et al: A Chinese family with lipoid proteinosis resulting from a homozygous missense mutation in the extracellular matrix protein 1 gene. J Eur Acad Dermatol Venereol 23:1336-1338 (2009).

Youssefian L, Vahidnezhad H, Daneshpazhooh M, Abdollahzadeh S, Talari H, et al: Lipoid proteinosis: phenotypic heterogeneity in Iranian families with c.507delT mutation in ECM1. Exp Dermatol 24:220-222 (2015).

Zhang R, Liu Y, Xue Y, Wang Y, Wang X, et al: Treatment of lipoid proteinosis due to the p.C220G mutation in ECM1, a major allele in Chinese patients. J Transl Med 12:85 (2014). 